\title{
多彩氧化铇薄膜的红外电致变色性能研究
}

\author{
武 琦 ${ }^{1,2}$, 丛 杉 $^{1}$, 赵志刚 ${ }^{1}$
}

(1. 中国科学院 苏州纳米技术与纳米仿生研究所, 苏州 215123 ; 2. 中国科学技术大学 纳米科学技术学院, 苏州 215123)

摘 要: 电致变色材料能够在可见-红外宽频谱范围内对吸收率、透过率、反射率和发射率进行动态调节, 是人工调 制材料光谱特征的有效手段。在实际应用场合, 对于色彩(可见光)与热辐射(红外线)可控调节的需求往往并存, 利 用单一电致变色器件实现可见、红外双波段的光学调制性能具有重要意义。现阶段对于电致变色材料的设计主要 集中于可见光谱的调节能力及颜色转变, 忽略了对材料红外光谱的探究。本研究选取具有法布里-铂罗(F-P)空腔结 构的多彩氧化铇薄膜进行可见-红外宽频谱电致变色探究, 具有 F-P 空腔结构的氧化铇薄膜材料在可见光区呈现多 样化、明亮的颜色, 再对多彩薄膜进行图案化设计, 即可得到具有各种图案的多彩薄膜, 并且在外加偏压的作用下 可以实现多样化的颜色转变。同时, 多彩氧化铇薄膜的红外反射率也可获得明显的可控调制效果, 在中波段 (Medium Wave, MW)3.5 $\mu \mathrm{m}$ 左右处不同颜色薄膜均可获得高于 $25.00 \%$ 的红外调制率。本研究表明, 多彩氧化钨薄膜 可以实现可见-红外宽频谱的分立、可控调节，在智能窗、热管理、辐射制冷等应用领域有巨大潜力，可达到光、 热性能协同调制的效果。

关 键 词: 多彩氧化铇; 电致变色; 红外调制

中图分类号: TQ174 文献标志码: A

\section{Infrared Electrochromic Property of the Colorful Tungsten Oxide Films}

\author{
WU Qi ${ }^{1,2}$, CONG Shan ${ }^{1}$, ZHAO Zhigang ${ }^{1}$
}

(1. Suzhou Institute of Nano-Tech and Nano-Bionics, Chinese Academy of Sciences, Suzhou 215123, China; 2. Nano Science and Technology Institute, University of Science and Technology of China, Suzhou 215123, China)

Abstract: Electrochromic materials are capable in a dynamically modulation over their absorption, transmission, reflection and emissivity covering the visible and infrared regions, which is recognized as one of the most effective strategies for an artificial control over the optical spectrum. In practical devices, the modulations for color (visible light) and heat (near-infrared irradiation) are both in demand, and thus it is of significant importance to achieve the dual-band control within single electrochromic device. However, the present design of electrochromic materials

收稿日期: 2020-08-13; 收到修改稿日期：2020-09-25; 网络出版时期: 2020-11-05

基金项目：国家自然科学基金(51772319，51772320，51972331); 江西省自然科学基金(20181ACB20011); 中国科学院对外 合作项目(121E32KYSB20190008); 江苏省六大人才高峰(XCL-170); 中国科学院青年创新促进会(2018356); 江 西省杰出青年基金(20192BCBL23027)

National Natural Science Foundation of China (51772319, 51772320, 51972331); Natural Science Foundation of Jiangxi Province (20181ACB20011); External Cooperation Program of the Chinese Academy of Sciences (121E32KYSB20190008); Six Talent Peaks Project of Jiangsu Province (XCL-170); Youth Innovation Promotion Association of Chinese Academy of Sciences (2018356); Outstanding Youth Fund of Jiangxi Province (20192BCBL23027)

作者简介: 武 琦(1997-), 女, 硕士研究生. E-mail: qwu2019@sinano.ac.cn WU Qi(1997-), female, Master candidate. E-mail: qwu2019@sinano.ac.cn

通信作者: 赵志刚, 研究员. E-mail: zgzhao2011@sinano.ac.cn ZHAO Zhigang, professor. E-mail: zgzhao2011@sinano.ac.cn 
mainly focuses on the adjustment ability of visible spectrum or color transformation, while leaving the modulation of infrared spectra often negligible. In this study, a multi-color tungsten oxide film with F-P cavity structure is selected to explore the wide-spectrum electrochromism of visible and infrared spectral regions. The tungsten oxide film material with F-P cavity structure presents diversified and bright colors in the visible region with various color patterns, and diversified color alternation can be realized under applied biases. At the same time, the infrared reflectance of colorful tungsten oxide films can also be modulated significantly, for example, infrared modulation rates higher than $25.00 \%$ can be obtained in the middle wavelength (around $3.5 \mu \mathrm{m}$ ) in the colored films. The research shows that the multi-color tungsten oxide film can realize the discrete and controllable adjustment of the visible and infrared spectral region in the wide spectrum, and has great potential in the application of smart window, thermal management, radiative cooling and other fields, which can even achieve the in-demand cooperative modulation of light and heat performance.

Key words: colorful tungsten oxide film; electrochromism; infrared modulation

电致变色材料, 是一种在外加电场的作用下通 过氧化还原反应使其颜色或光学特性(反射率、透过 率、吸收率等)发生可逆变化的材料, 在节能窗、显 示屏、热管理、辐射制冷等多个领域具有广泛的应 用前景, 成为近年来学术界和产业界共同关注的研 究热点 ${ }^{[1-4]}$ 。值得注意的是, 传统的电致变色材料尤 其是无机电致变色材料的设计, 大多是追求对可见 光谱的调节能力 ${ }^{[5-7]}$, 而针对红外光谱调控的研究 相对较少。众所周知, 红外光所占据的波段分为四 部分, 即近红外、中红外、远红外和极远红外 ${ }^{[8]}$ 。其 中中红外光通常被定义为波长处于 $2.5 \sim 25 \mu \mathrm{m}$ 的电 磁波，其不仅可用于分子含量的检测和分子类型的鉴 定，还可以通过红外热成像用于体温监测等领域 ${ }^{[9-11]}$ 。 因此研究无机电致变色材料在红外光区间的电致变 色调控特性具有重要意义。

氧化铇作为最经典的无机电致变色材料, 具有 良好的循环稳定性及较高的光学调制率等特性, 一 直是电致变色领域的研究热点之一 ${ }^{[12]}$ 。然而, 氧化 铇作为电致变色材料仍然存在一些急需解决的关键 科学问题。例如, 在可见光波段的光谱调制中, 氧化 铇颜色变化十分单一, 通常只能实现无色和蓝色两 种状态之间的切换 ${ }^{[13-14]}$; 又如, 在基于氧化铇材料 的电致变色器件中, 在宽频谱范围内实现对光/热的 分立调制也是一大挑战 ${ }^{[15]}$ 。最近, 本课题组在薄膜 氧化铇材料中引入金属薄层, 通过构筑法布里一珀 罗谐振腔结构(一种由两个平行平面反射镜组成的 光学谐振腔 $)^{[16-19]}$, 使用全无机材料实现了包括红 橙黄绿青蓝紫在内的近全彩展示; 进一步通过外加 偏压, 实现对各色系中色调、饱和度和明度更为丰 富、精细地调控 ${ }^{[20-21]}$ 。通过引入结构色实现丰富色 彩间的可控、可逆切换是无机电致变色材料领域的
一大突破, 将大大拓展无机电致变色材料的应用范 畴。在实际应用场合, 对于色彩(可见光)与热辐射 (红外线)可控调节的需求往往并存, 然而目前的电 致变色器件在此领域的应用报道几乎为空白。可见 光与红外线是波长不同的电磁波, 从光学结构材料 角度看有相似的调制规律; 这种基于物理色与化学 色相结合的光谱调制手段同样会对材料的红外吸收/ 反射性质产生影响，从而实现宽频谱的有效调制， 这也是多彩氧化物薄膜内在的优势之一。本课题组 前期关于多彩氧化铇薄膜的研究工作止步于实现可 见光区的色彩的精细调控, 而对于其在中-长红外 宽频谱范围的可控调节还有待探索。

基于上述研究背景, 本工作将探究电致变色过程 中, 多彩氧化铇薄膜在红外波段的光谱调制性能, 主 要包括中波段(Medium Wave, MW, 3 5 $\mu \mathrm{m}$ )及长波段 (Long Wave, LW, 8 12 $\mu \mathrm{m}$ ) 两个波段范围 ${ }^{[22]}$ 。通过材 料物性表征与光谱测试手段, 研究材料结构与光谱 调制性能之间的构效关系; 通过薄膜组成结构优化, 在多彩氧化铇薄膜中实现可见-红外宽频谱共同调 制的能力, 进而实现多彩氧化铇光热性能的协同调 控，并初步探索多彩氧化铇在红外调制方向的应用。

\section{1 实验方法}

\section{1 材料制备}

采用磁控溅射法制备多彩氧化铇薄膜。首先将 硅片、PET 基底或其他柔性基底剪裁成合适大小 $(1.5 \mathrm{~cm} \times 3.5 \mathrm{~cm})$, 然后将基底放在模具上固定于磁 控溅射设备腔体中, 抽真空后按照设定程序及参数 进行磁控溅射。设定程序为: 以压力 $0.3 \mathrm{~Pa}$ 、功率 $200 \mathrm{~W}$ 沉积底层 $\mathrm{W}$ 金属层(约 $100 \mathrm{~nm}$ ), 以功率 
$100 \mathrm{~W}$ 、氩氧比 $27: 81$ 沉积顶层 $\mathrm{WO}_{3}$ 层 $(130 \sim 240 \mathrm{~nm})$, 祄底转速为 $20 \mathrm{r} / \mathrm{min}$ 。两层薄膜材料均由 $\mathrm{W}$ 靶材制 得, 氧气和氩气纯度都为 $99.99 \%$ 。

\section{2 材料表征与性能测试}

采用椭圆偏振仪测试镀在硅片上的多彩氧化铇 薄膜的厚度; 采用 CHI760 电化学工作站以三电极 体系实现电致变色过程, 其中, 电致变色材料作为 工作电极, 铂丝作为对电极, $\mathrm{Ag} / \mathrm{AgCl}$ 电极(电解液 为饱和 $\mathrm{KCl}$ 溶液)作为参比电极, $1 \mathrm{~mol} / \mathrm{L} \mathrm{Al}\left(\mathrm{ClO}_{4}\right)_{3} /$ $\mathrm{PC}$ 溶液作为电致变色电解液; 采用紫外-可见分光 光度计(V660, JASCO)测试可见光区域(350 800 nm) 薄膜的反射率曲线; 采用傅里叶红外光谱仪 (Nicolet 6700)测试中红外区域薄膜的反射率曲线, 测试波长为 $2.5 \sim 25 \mu \mathrm{m}$; 采用红外热像仪(TiX580, 美国 Fluke 公司, 红外光谱范围: 7.5 14 $\mu \mathrm{m}$ )拍摄红 外图像。

测试薄膜的反射率曲线, 需要先用电化学工作 站对工作电极施加一定电压进行电致变色处理, 然 后用乙醇冲洗材料表面残余有机电解液，最后再分 别进行可见光区、中红外区的反射率测试。

\section{2 结果与讨论}

\section{1 薄膜可见光区的色彩调制范围}

在柔性基底 PET 或 PET-ITO 上依次溅射约 $100 \mathrm{~nm}$ 的金属 $\mathrm{W}$ 层和 130 240 nm 的 $\mathrm{WO}_{3}$ 层, 使薄 膜内部形成非对称性的 F-P 空腔结构(图 1(a)), 根据 断面 SEM 照片(图 1(b))也可以很清楚地看到这种 $\mathrm{W} / \mathrm{WO}_{3}$ 双层结构, 利用结构色在氧化铇材料中实 现多彩色展示。通过精细控制 $\mathrm{WO}_{3}$ 层的厚度, 可以 调制相应的共振波长, 从而实现对可见光区域色彩 的连续调制。通过磁控溅射法制得的不同色彩氧化 铇薄膜样品如图 1(c)所示, 几乎包含黄橙红紫蓝青 绿等全部基本色, 且各色彩有着较高的饱和度。进 一步, 通过对多彩薄膜进行图案化设计, 得到具有 各种生动动物形象的彩色图案, 体现了这种多彩薄 膜结构的稳定性与可加工性。根据 F-P 空腔结构中 物理色的产生原理, 所得薄膜的光谱性质与其中氧 化铇介电层的厚度密切相关 ${ }^{[20]}$ 。分析其反射率曲线 (图 1(d)) 可以得知, 随着 $\mathrm{WO}_{3}$ 厚度的增加, 反射谷 的位置从 462 到 $710 \mathrm{~nm}$ 发生了较大的红移, 而在短 波长区间(363 446 nm)出现了新的反射谷。因此, 在 所得 F-P 空腔结构中, 仅通过对氧化铇层厚度的控 制，即可获得可见谱区反射谷的连续调制，从而实 现基本色的展示。

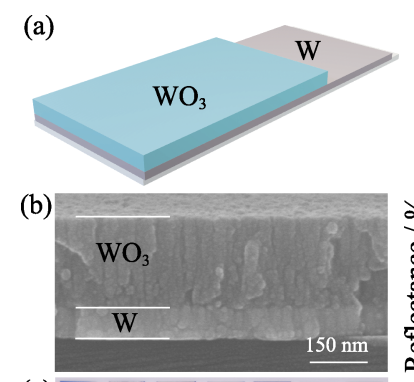

(c)

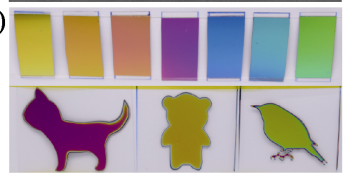

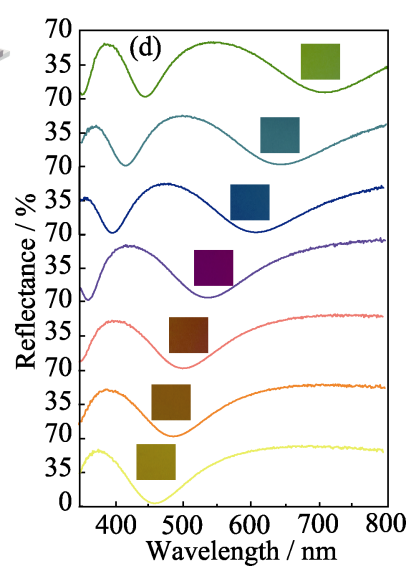

图 1 多彩膜的结构示意图(a), 断面 SEM 照片(b), 实拍薄 膜样品和图案化样品(c), 以及七种颜色的反射率曲线(d)

Fig. 1 Schematic illustration of the colorful film (a), crosssectional SEM image (b), optical images of the electrochromic electrodes with different colors and the patterned samples (c), and reflectance spectra of electrode films with seven colors (d)

通过构筑的 F-P 空腔结构, 仅使用无机薄膜材 料即可实现丰富色彩的静态展示, 进一步, 将上述 薄膜结构与氧化铇的电致变色特性相结合, 通过外 加偏压定量控制电子/离子在氧化铇材料晶格中的 可逆插入/脱出, 实现了对上述结构中氧化铇薄层光 学性质 ( $n 、 k$ 值)的精细调制, 即在上述物理色的基 础上, 通过化学色极大地丰富材料的色调, 并赋予 其动态调节的能力(图 2(a))。此过程发生的代表性化 学反应如下:

$$
\mathrm{WO}_{3}+x \mathrm{Al}^{3+}+3 x \mathrm{e}^{-} \leftrightarrow \mathrm{Al}_{x} \mathrm{WO}_{3}
$$

当氧化铇材料很厚并且没有金属反射层存在时， 薄膜没有结构色产生, 外观颜色都属于本征的化学 色。在这种条件下, 氧化铇在不同施加电压下发生 可逆氧化还原反应并在氧化铇和铇青铜材料间切 换。两种材料的光学常数 $(n 、 k)$ 不同, 二者之间只 是化学色的调制, 因此得到的颜色只有透明到蓝色 的转变; 而当选择合适金属反射层并调节氧化铇材 料到适当厚度时, 薄膜除了表现出材料本征的化学 色，这种特殊结构中的 F-P 谐振腔效应还会显现结 构色(物理色)。不仅如此, 介质材料的光学常数 ( $n$ 、 $k)$ 还是结构色重要的影响参数, 而在不同电压下调 节氧化铇材料的光学常数 $(n 、 k)$, 使 $\mathrm{W} / \mathrm{WO}_{3}$ 这种结 构最终可以表现出物理色和化学色叠加颜色的调 制。分别选取黄色、紫色、绿色三种颜色的多彩氧 化铇薄膜作为代表, 深入分析电致变色电压对可见 光区反射率曲线的影响, 如图 $2(b \sim d)$ 所示。随着外 加负压的升高, 黄色薄膜逐渐变为黄绿色、绿色和 墨绿色, 紫色薄膜逐渐变为红色、棕色和茶色, 绿色 

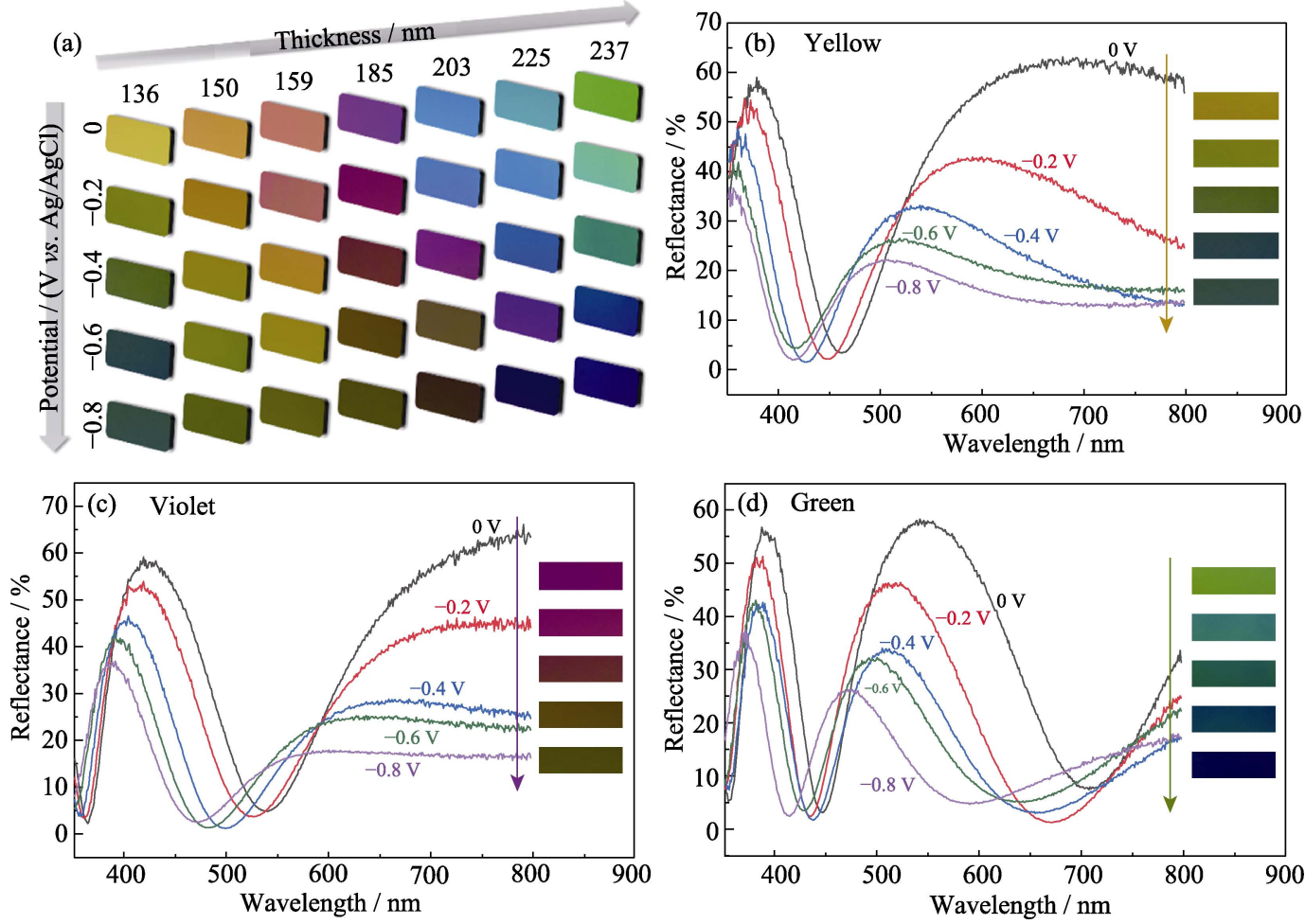

图 2 多彩氧化铇薄膜在不同电压下电致变色的光学图片(a)和黄色、紫色、绿色三种薄膜在不同电压下反射率曲线变化(b-d) Fig. 2 Optical images of electrochromic films at different applied potentials (a), variation of reflectance spectra of yellow, purple and green films at different applied potential (b-d)

薄膜则逐渐变成蓝色、深蓝色和紫色; 这种直观的 颜色变化同样可由反射曲线清晰地反映, 三种颜色 薄膜的反射率均逐渐减小, 反射峰也逐渐蓝移, 这 和变色后多彩氧化铇的视觉颜色逐渐向深色系变化 的规律相一致，同时也显示出在可见光区域控制电 致变色电压可以对不同颜色实现精细调制。

\section{2 薄膜中红外光区的调制范围}

为了研究多彩氧化铇薄膜在红外波段的调制性 能, 首先采用了 FT-IR 红外光谱仪在 3 13 $\mu \mathrm{m}$ 区域 内进行反射率测试 ${ }^{[11]}$, 测试前先用金镜进行校准, 再对 $\mathrm{CO}_{2}$ 和 $\mathrm{H}_{2} \mathrm{O}$ 进行校正, 排除测试环境对薄膜的 干扰, 以确保数据的准确性。图 3 为不同颜色的多 彩氧化铇薄膜的红外反射率曲线, 在没有外加电场 作用的情况下，具有相同厚度金属 $\mathrm{W}$ 层的薄膜在测 试范围内红外反射率总体趋于较高的水平; 随着 $\mathrm{WO}_{3}$ 厚度的增加, 薄膜在 3 13 $\mu \mathrm{m}$ 光谱区间的反射 率逐渐降低, 主要是由于氧化物 $\mathrm{WO}_{3}$ 层对金属 $\mathrm{W}$ 层的高反射有阻挡作用。但薄膜在中波段 $(\mathrm{MW}$, $3 \sim 5 \mu \mathrm{m})$ 的红外反射率仍达到平均 $75 \%$ 左右, 长波 段( $(\mathrm{LW}, 8 \sim 12 \mu \mathrm{m}$ ) 的红外反射率则平均在 $80 \%$ 左右。 另外, 在红外光谱图中发现 $10.5 \mu \mathrm{m}\left(952 \mathrm{~cm}^{-1}\right)$ 附近 有一个红外特征峰, 该红外特征峰归属于 $\mathrm{WO}_{3}$ 中的 $\mathrm{W}=\mathrm{O}$ 键的不对称伸缩振动, 下面也会提到对该峰 的相关验证。

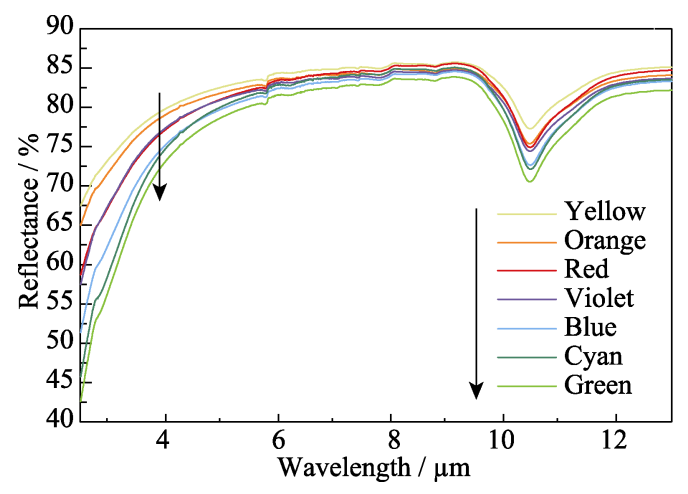

图 3 多彩氧化铇薄膜在中红外区 $(3 \sim 13 \mu \mathrm{m})$ 的反射率曲线 Fig. 3 Reflectance spectra of the tungsten oxide film in the mid-infrared region $(3-13 \mu \mathrm{m})$

随机选择黄色、紫色、绿色薄膜施加不同负压 进行电致变色处理, 探究不同电致变色电压对薄膜 红外反射率的影响, 如图 4(a c) 所示。从图中可以 看出, 在电致变色过程中, 三种薄膜的红外反射率 展现出相似的规律, 即随着外加偏压由 $0 \mathrm{~V}$ 改变至 $-0.8 \mathrm{~V}$, 薄膜的红外反射率逐渐减小, 证实了多彩 氧化铇薄膜在电致变色的离子/电子插入条件下对 红外光谱反射性能的调制能力。为了定量对比不同 颜色薄膜之间的红外反射率调制差别, $\Delta R$ 表示电致 变色处理前后薄膜红外反射率的差值, 如表 1 所示。 在中波段 $(\mathrm{MW}, 3.5 \mu \mathrm{m})$ 左右, 不同颜色薄膜均可获 得较大的红外调制率, 其中黄色薄膜的红外调 

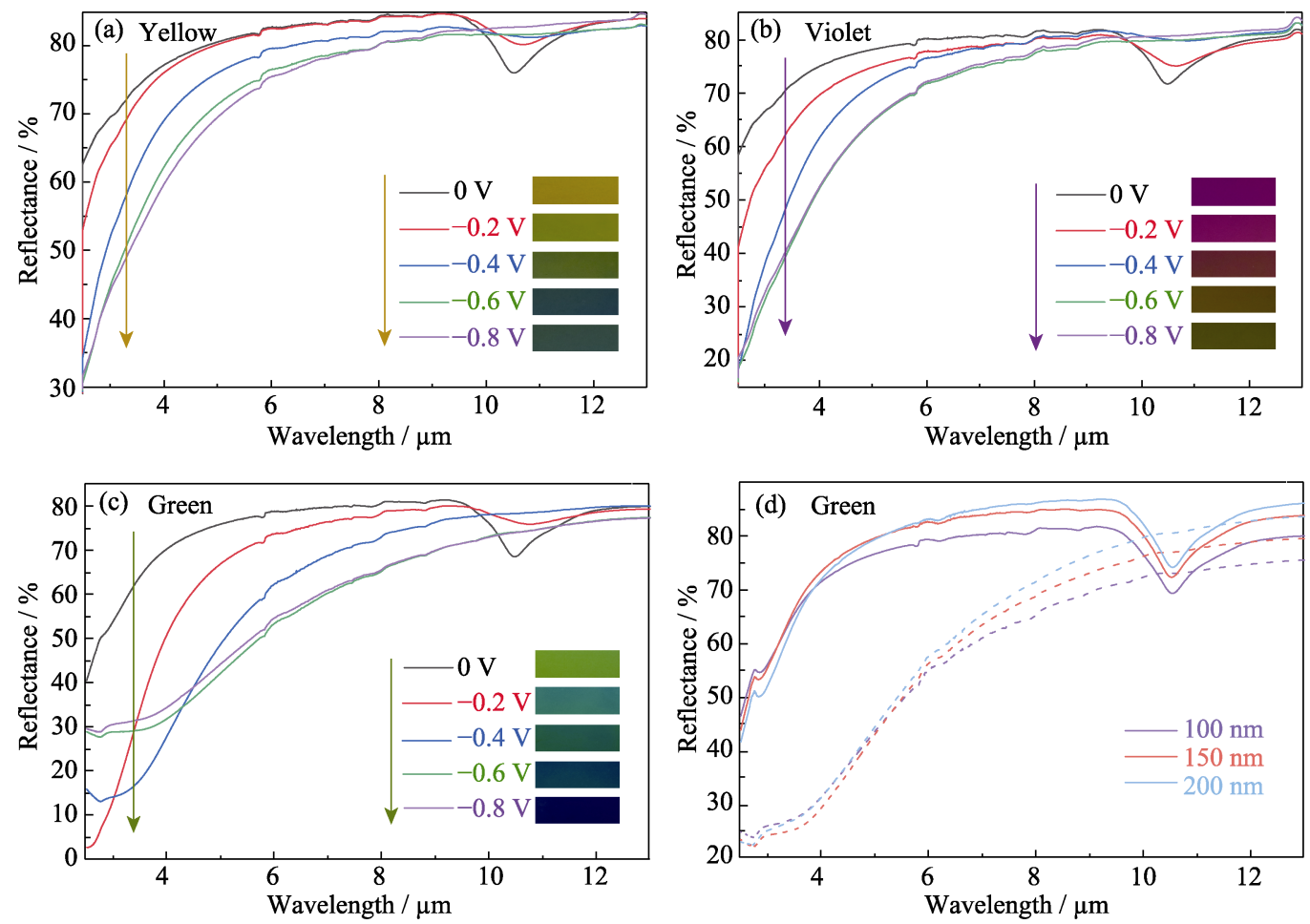

图 4 黄色、紫色、绿色三种薄膜在不同电压下的中红外反射率曲线 $(\mathrm{a} \sim \mathrm{c})$ 和不同 $\mathrm{W}$ 厚度的绿色薄膜的红外反射率曲线 $(\mathrm{d})$

Fig. 4 Variation of mid-infrared reflectance spectra of yellow, purple and green films at different applied potentials (a-c), mid-infrared reflectance spectra of green films with different $\mathrm{W}$ thicknesses (d)

Solid and dotted lines in (d) are the samples before and after coloration, respectively, wherein the applied voltage is fixed at $-0.6 \mathrm{~V}$

表 1 薄膜在 MW 和 $\mathbf{L W}$ 的反射率调制

Table 1 Reflectance modulation of films in MW and LW

\begin{tabular}{ccc}
\hline Color & Band & $\Delta R$ \\
\hline Yellow & MW & $25.61 \%$ \\
& LW & $6.67 \%$ \\
Violet & MW & $33.68 \%$ \\
& LW & $8.91 \%$ \\
Green & MW & $46.00 \%$ \\
& LW & $15.39 \%$ \\
\hline
\end{tabular}

制率为 $25.61 \%$, 紫色薄膜的红外调制率为 $33.68 \%$, 绿色薄膜的红外调制率约为 $46.00 \%$, 说明不同结构 薄膜的红外调制能力存在较大的差别，而这种差别 可能与 $\mathrm{WO}_{3}$ 的厚度有一定关系。相比之下，在长波 段(LW, 8 12 $\mu \mathrm{m})$, 几种薄膜的红外调制率均较小, 仅为 $5 \%$ 15\%左右，这与文献报道的结果相一致， 即长波段的红外调制率均低于中波段 ${ }^{[22]}$ 。为了探究 $\Delta R$ 与薄膜结构之间的联系, 通过计算研究了薄膜 结构与红外调制能力之间的构效关系, 发现 $\mathrm{WO}_{3}$ 层厚度与电致变色过程中插入电量是决定其红外 反射率的两大因素; 且无论是中波段还是长波段, 随着 $\mathrm{WO}_{3}$ 厚度的增加, $\Delta R$ 均呈现增大的趋势。

根据基尔霍夫定律 ${ }^{[23-24]}, \varepsilon=A=1-T-R$ 。式 中 $\varepsilon$ 为材料的发射率, $A$ 为吸收率, $T$ 为透射率, $R$ 为
反射率。

当材料的透过率几乎为零时, $\varepsilon=A=1-R$, 材 料的红外反射率主要由材料在特定波长下的红外吸 收来决定。

由于 $\mathrm{WO}_{3}$ 的红外吸收在一定程度上是来自于 电致变色过程中离子嵌入后的极化子振动吸收 ${ }^{[25]}$ 。 因此，嵌入的铝离子量越多，其红外吸收就越高, 发射率(或反射率)调制范围也就越大。

另外, 研究中发现多彩氧化铇薄膜的金属 W 层 对可见光反射光谱没有影响，即结构色不受金属层 的影响 ${ }^{[20]}$; 但金属层却会直接影响多彩膜对红外光 谱的调制能力。探究具有不同 $\mathrm{W}$ 层厚度的绿色薄膜 的红外光区光学性能, 如图 4(d)所示。随着 W 层厚 度的增加, 绿色薄膜的红外反射率逐渐增大, 而在 施加相同负压后, 薄膜的反射率依旧呈现下降趋 势。通过计算 $\mathrm{W}$ 厚度分别为 $100 、 150$ 和 $200 \mathrm{~nm}$ 的 绿色薄膜变色前后的 $\Delta R$ 数值, 发现其在 $4 \mu \mathrm{m}$ 波段 处分别为 $40 \% 、 44 \%$ 和 $41 \%$, 而在 $8 \mu \mathrm{m}$ 波段处分别 为 $16 \% 、 16 \%$ 和 $15 \%$, 这说明通过优化金属层 $\mathrm{W}$ 厚 度虽然能提高多彩膜的初始红外反射率，但是对红 外反射率的调制范围影响不大。

值得注意的是, 随着外加负压的增大, 在红外 光谱图中 $10.5 \mu \mathrm{m}\left(952 \mathrm{~cm}^{-1}\right)$ 附近的红外特征峰逐渐 
消失, 由于该峰归属于氧化物材料中 $\mathrm{W}=\mathrm{O}$ 键的不 对称伸缩振动 ${ }^{[26]}$, 其消失可能是由于 $\mathrm{Al}^{3+}$ 的插入导 致的, F-P 空腔结构中的 $\mathrm{WO}_{3}$ 层逐渐变成铇青铜结 构的 $\mathrm{Al}_{x} \mathrm{WO}_{3}$, 而铇青铜结构具有较强的红外吸收/ 屏蔽性能, 这也是多彩氧化铇薄膜具备良好红外调 制性能的主要原因。

\section{3 实际应用探索}

基于上述薄膜器件的多彩展示与红外调制性能, 探究了其在宽频谱调控方面的应用。作为功能演示, 分别考察了不同颜色的多彩膜(图 5(a)), 以及绿色 多彩膜在不同负压变色后(图 5(b)) 的红外屏蔽性能。 实验过程中, 将薄膜置于 $60{ }^{\circ} \mathrm{C}$ 的加热板上, 用红 外热成像仪分别记录其红外图像。从图 5(a)中可以 观察到作为对照组的基底 PET 呈现与加热板相同的 颜色, 证实了 PET 基底并无红外屏蔽性能; PET/W 与 $\mathrm{PET} / \mathrm{WO}_{3}$ 对照组表面温度分别为 43.5 和 $52.2{ }^{\circ} \mathrm{C}$, 红外成像视场下呈现与热板及背景不相同的颜色, 说明金属 $\mathrm{W}$ 层或 $\mathrm{WO}_{3}$ 本身具有一定的红外屏蔽能
力, 但显然不适合实际应用。相比之下, 不同颜色的 多彩膜实验组在红外成像仪中观察到的颜色十分相 近, 且与环境背景几乎一致, 说明将原本没有红外 屏蔽效果的两种材料——金属 $\mathrm{W}$ 与 $\mathrm{WO}_{3}$ 构成 F-P 空腔结构以后, 可以赋予复合材料良好的红外屏蔽 性能, 这与图 3 中多彩膜的红外反射率测试结果相 一致。从图 5(b c) 中可以看出, 在对绿色薄膜分别施 加不同的负压变色后, 在光学颜色发生变化的同时, 其表面热辐射效果也得到进一步调制, 证实了薄膜 结构的宽频谱独立调制能力。由图 5(b)可见, 当外加 负压分别为 $-0.4 、-0.6$ 和 $-0.8 \mathrm{~V}$ 时, 薄膜的红外图像 中间有一条明显的分界线, 线下对应薄膜的变色部 分, 而线上对应未变色部分; 其中, 变色部分相比 于未变色薄膜的温度低 $1 \sim 2{ }^{\circ} \mathrm{C}$, 这与图 5(d)中薄膜 电致变色处理后的红外反射率下降趋势相一致。以 上结果证实了通过构筑F-P 空腔结构, 在所得多色彩 薄膜中获得了较强的红外屏蔽性能, 并可通过外加 偏压实现动态调制，具备应用于宽频谱调控的潜力。

(a)

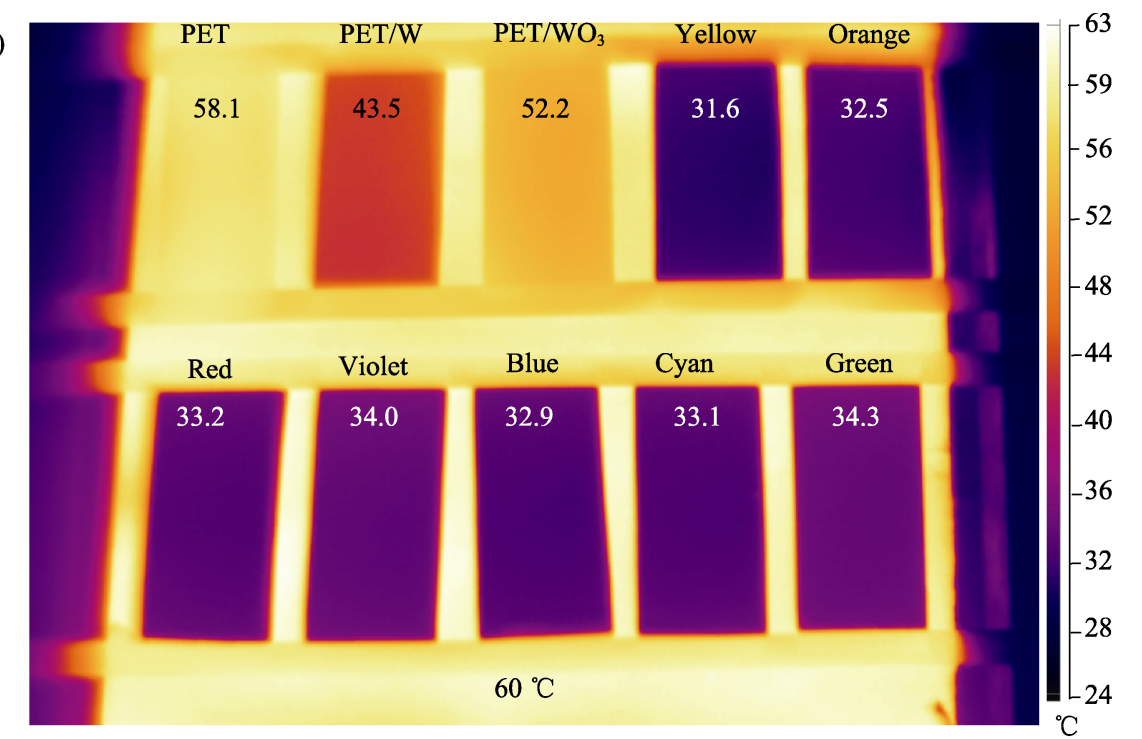

(b)

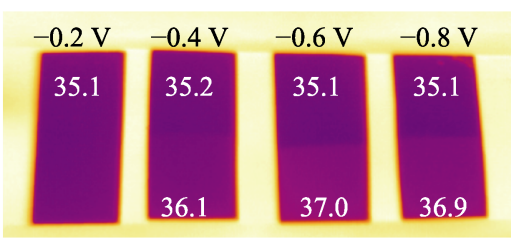

(c)
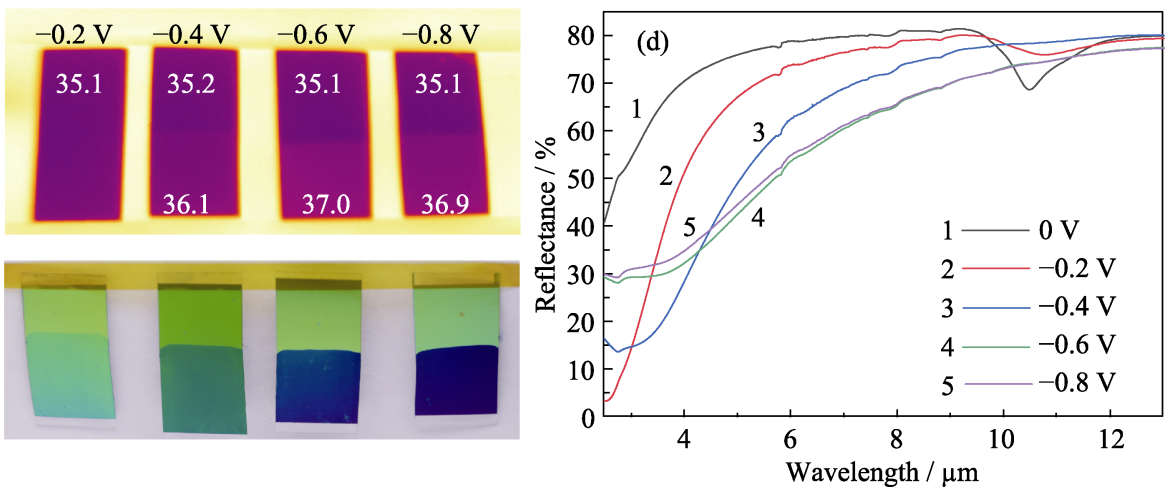

图 5 不同颜色的氧化铇薄膜的红外热成像图像(a), 对绿色薄膜加不同负压的红外热成像图像(b)、 光学图像(c)及对应的反射率曲线变化(d)

Fig. 5 Infrared thermal imaging of tungsten oxide films of different colors (a), infrared thermal imaging photos of green films with different negative potentials (b), optical images (c) and their corresponding reflectance curve changes (d) 


\section{3 结论}

基于 F-P 空腔结构，通过构筑金属 $\mathrm{W}$ 与氧化铇 的叠层薄膜结构, 在无机电致变色材料中获得了近 全彩的多色展示, 进一步探究了多彩氧化铇薄膜的 微观结构与光谱调制性能之间的构效关系, 并研究 了外加偏压条件下, 多彩膜对可见-中红外波段宽 频谱调制能力。实验表明, 所得叠层薄膜结构能够 实现几乎全彩色展示, 并且在改变电致变色的条件 下，呈现更多样化的颜色转换; 金属 $\mathrm{W}$ 层厚度对复 合薄膜的色彩无影响，但会影响材料的红外反射率; 本研究中的多彩氧化铇薄膜具有较强的红外屏蔽能 力, 其颜色和中红外区的反射率均可随外加偏压改 变, 具备可控调节的能力, 在热管理、辐射制冷等领 域有很大的应用前景。

\section{参考文献:}

[1] GRANQVIST C G. Handbook of Inorganic Electrochromic Materials. Amsterdam: Elsevier, 1995: 9-13.

[2] MORTIMER R J, ROSSEINSKY D R, MONK P M S. Electrochromic Materials and Devices. New York: Wiley-VCH Verlag GmbH \& Co. KGaA. 2015: 3-33.

[3] MORTIME R J. Electrochromic materials. Annual Review of Materials Research, 2011, 41(1): 241-268.

[4] WANG ZHEN, WANG XIAO-YU, CONG SHAN, et al. Fusing electrochromic technology with other advanced technologies: a new roadmap for future development. Materials Science and Engineering: R-Reports, 2020, 140: 100524-1-26.

[5] LI NA, LI YAO, YIN YA-DONG, et al. Dynamically switchable multicolor electrochromic films. Small, 2019, 15(7): 1804974-1-7.

[6] XIAO LI-LI, LÜ YING, LIU XING-YUAN, et al. $\mathrm{WO}_{3}$-based electrochromic distributed Bragg reflector: toward electrically tunable microcavity luminescent device. Advanced Optical Materials, 2017, 6(1): 1700791-1-8.

[7] LI HAI-ZENG, FIRBY C J, ELEZZABI A Y, et al. Rechargeable aqueous electrochromic batteries utilizing Ti-substituted tungsten molybdenum oxide based $\mathrm{Zn}^{2+}$ ion intercalation cathodes. Advanced Materials, 2019, 31(15): 1807065-1-9.

[8] ROGALSKI A, CHRZANOWSKI K. Infrared devices and techniques. Opto-Electronics Review, 2002, 10(2): 111-136.

[9] XU CHENG-YI, STIUBIANU G T, GORODETSKY A A. Adaptive infrared-reflecting systems inspired by cephalopods. Science, 359(6383): 1495-1500.

[10] PENG LIANG, LIU DONG-QING, ZU MEI, et al. A multilayer film based selective thermal emitter for infrared stealth technology. Advanced Optical Materials, 2018, 6(23): 1801006-1-8.

[11] LYU J, LIU ZENG-WEI, ZHANG XUE-TONG, et al. Nanofibrous Kevlar aerogel films and their phase-change composites for highly efficient infrared stealth. ACS Nano, 2019, 13: 2236-2245.

[12] CAI GUO-FA, WANG JIANG-XIN, LEE P S. Next-generation multifunctional electrochromic devices. Accounts of Chemical Research, 2016, 49(8): 1469-1476.

[13] CAI GUO-FA, CUI MENG-QI, LEE P S, et al. Ultra-large optical modulation of electrochromic porous $\mathrm{WO}_{3}$ film and the local monitoring of redox activity. Chemical Science, 2016, 7(2): 1373-1382.

[14] CONG SHAN, GENG FENG-XIA, ZHAO ZHI-GANG, et al. Single-crystalline tungsten oxide quantum dots for fast pseudocapacitor and electrochromic applications. Advanced Materials, 2014, 26(25): 4260-4267.

[15] WANG ZHEN, ZHANG QING-ZHU, ZHAO ZHI-GANG, et al. Using intrinsic intracrystalline tunnels for near-infrared and visiblelight selective electrochromic modulation. Advanced Optical Materials, 2017, 5(11): 1700194-1-6

[16] ZHAO JIAN-CUN, LEI DANG-YUAN, YU YI-TING, et al. Defining deep-subwavelength-resolution, wide-color-gamut, and large-viewing-angle flexible subtractive colors with an ultrathin asymmetric Fabry-Perot lossy cavity. Advanced Optical Materials, 2019, 7(23): 1900646-1-8.

[17] YANG ZHENG-MEI, ZHOU YAN-MING, DUAN HUI-GAO, et al. Reflective color filters and monolithic color printing based on asymmetric Fabry-Perot cavities using nickel as a broadband absorber. Advanced Optical Materials, 2016, 4(8): 1196-1202.

[18] YANG ZHENG-MEI, JI CHEN-GANG, LIU DONG, et al. Enhancing the purity of reflective structural colors with ultrathin bilayer media as effective ideal absorbers. Advanced Optical Materials, 2019, 7(21): 1900739-1-9.

[19] LEE K T, HAN S Y, PARK H J. Omnidirectional flexible transmissive structural colors with high-color-purity and highefficiency exploiting multicavity resonances. Advanced Optical Materials, 2017, 5(14): 1700284-1-9.

[20] WANG ZHEN, WANG XIAO-YU, ZHAO ZHI-GANG, et al. Towards full-colour tunability of inorganic electrochromic devices using ultracompact Fabry-Perot nanocavities. Nature Communications, 2020, 11(1): 302-1-9.

[21] CHEN JIAN, WANG ZHEN, ZHAO ZHI-GANG, et al. Fabry-Perot cavity-type electrochromic supercapacitors with exceptionally versatile color tunability. Nano Letters, 2020, 20(3): 1915-1922.

[22] SAUVET K, SAUQUES L, ROUGIER A. IR electrochromic $\mathrm{WO}_{3}$ thin films: from optimization to devices. Solar Energy Materials and Solar Cells, 2009, 93(12): 2045-2049.

[23] SALISBURY J W, WALD A, D'ARIA D M. Thermal-infrared remote sensing and Kirchhoff's law 1. laboratory measurements. Journal of Geophysical Research: Solid Earth, 1994, 99(B6): 11897-11911.

[24] KORB A R, SALISBURY J W, D'ARIA D M. Thermal-infrared remote sensing and Kirchhoff's law: 2. field measurements. Journal of Geophysical Research: Solid Earth, 1999, 104(B7): $15339-15350$

[25] ZHANG XIANG, TIAN YAN-LONG, LI YAO, et al. Preparation and performances of all-solid-state variable infrared emittance devices based on amorphous and crystalline $\mathrm{WO}_{3}$ electrochromic thin films. Solar Energy Materials and Solar Cells, 2019, 200: 109916-1-6.

[26] CHONG S V, INGHAM B, TALLON J L. Novel materials based on organic-tungsten oxide hybrid systems I: synthesis and characterization. Current Applied Physics, 2004, 4: 197-201. 